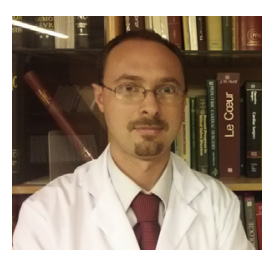

\section{REPLY: THE CHALLENGES OF COMPREHENSIVE ASSESSMENT IN ISCHEMIC CARDIOMYOPATHY Reply to the Editor:}

We are increasingly confronted with (and increasingly accept for surgery) candidates for coronary artery bypass grafting (CABG) presenting with severely reduced left ventricular ejection fraction (LVEF). In the Rennes University prospective database including 8276 isolated CABG operations performed between 2000 and 2019, the rate of patients with preoperative $\mathrm{LVEF} \leq 35 \%$ increased from $0.9 \%$ in 2000 to $5 \%$ in 2019 . Among these patients, the observed operative mortality was $2.9 \%$. In their article recently published in the Journal, Omer and associates ${ }^{1}$ not only confirm the known association between depressed LVEF and increased early mortality after cardiac surgery, but also suggest that rescued major postoperative complications have an unfavorable impact on late survival regardless of the severity of left ventricular (LV) dysfunction. The prediction of outcomes in these patients has often been puzzling, with the demonstration of myocardial viability often considered the cornerstone of discrimination between the futility and usefulness of myocardial revascularization surgery despite increased risk. In a recent important article, Drs Fukunaga and $\mathrm{Rao}^{2}$ emphasized that significant LV dilation predicted early mortality and morbidity after coronary surgery. They then formulated the hypothesis that the interaction between LV dilation and LV ventricular dysfunction could better predict the long-term effect of rescued early postoperative complications on late survival, ${ }^{3}$ possibly by identifying cases where too-far advanced myocardial disease will compromise the patients' physiological capability to overcome major complications and a prolonged intensive care unit/hospital stay. The singlecenter nature of their series guarantees uniformity in decision making criteria and in patient evaluation.

Appropriate decision making in patients with advanced ischemic cardiomyopathy can be compared with an equation containing several variables. Addressing only one variable is insufficient to solve the problem and leads to suboptimal outcomes. For example, myocardial viability is an important element, yet is insufficient to predict survival when it is the sole parameter addressed. ${ }^{4}$ The same could be said for LVEF and LV end-diastolic diameter. The degree of LV dilation can be viewed as a component of a more complex phenomenon, LV remodeling. In that sense, the suggestion by Fukunaga and Rao is important and certainly warrants further exploration. It remains to be clarified whether more in-depth analyses can be done through the VASQIP database, which includes a huge number of observations but also features limited data on LV morphology and viability. VASQIP is also contributed to by numerous many institutions with different protocols for patients' evaluation and decision making, as well as variable local expertise in heart failure management.

A parallel might be established between the current subject and the MITRA-FR/COAPT debate. ${ }^{5}$ Such debate underscores the central importance of appreciation of myocardial disease versus secondary mitral regurgitation (MR) in determining patients' outcome. Treating proportionate mitral insufficiency in patients with an excessively dilated LV is scarcely beneficial, as advanced myocardial remodeling finally dictates a continued evolution through heart failure. Should we address a proportionate/disproportionate notion not only for secondary MR, but also for LV remodeling in the context of advanced coronary disease amenable to CABG? When disproportionate LV remodeling would be dismal for early/late overall prognosis and LV recovery after CABG (unfavorable risk/benefit balance), other treatment options should be discussed. The assessment of this status should rely on the interaction of multiple parameters-not only LVEF and LV diameter, but also extension of viable myocardium and LV sphericity index, ${ }^{6}$ all of which are variables in the equation. Such an approach is ambitious to structure, possibly sufficiently complex to warrant application of artificial intelligence to imaging data. The current debate suggests that patients with advanced ischemic cardiomyopathy and an option for revascularization should be discussed and treated in reference centers where multidisciplinary management of heart failure is customary and all treatment options, including the various forms of mechanical circulatory support, are available.

\section{Amedeo Anselmi, $M D, P h D$ Division of Thoracic and Cardiovascular Surgery Pontchaillou University Hospital Rennes, France}

\section{References}

1. Omer S, Adeseye A, Jimenez E, Cornwell L, Massarweh N. Low left ventricular ejection fraction, complication rescue, and long-term survival after coronary artery bypass grafting. J Thorac Cardiovasc Surg. 2020. https://doi.org/10.1016/ j.jtcvs.2020.03.040

2. Fukunaga N, Pinto Ribeiro RV, Lafreniere-Roula M, Manlhiot C, Badiwala M, Rao V. Left ventricular size and outcomes in patients with left ventricular ejection fraction less than 20. Ann Thorac Surg. 2020. https://doi.org/10.1016/j.athoracsur.2020.01.005

3. Fukunaga N, Rao V. The importance of left ventricular size. J Thorac Cardiovasc Surg. 2022; 163:e183.

4. Panza J, Ellis A, Al-Khalidi H, Holly T, Berman D, Oh J, et al. Myocardial viability and long-term outcomes in ischemic cardiomyopathy. $N$ Engl J Med. 2019;381:739-48.

5. Leurent G, Anselmi A, Donal E. Clip therapy for secondary mitral regurgitation: the beginning of a long story? Acta Cardiol. 2020;75:186-8. 
The author reported no conflicts of interest.

The Journal policy requires editors and reviewers to disclose conflicts of interest and to decline handling or reviewing manuscripts for which they may have a conflict of interest. The editors and reviewers of this article have no conflicts of interest.

6. Choi JO, Daly RC, Lin G, Lahr BD, Wiste HJ, Beaver TM, et al. Impact of surgical ventricular reconstruction on sphericity index in patients with ischaemic cardiomyopathy: follow-up from the STICH trial. Eur J Heart Fail. 2015;17: 453-63.

https://doi.org/10.1016/j.jtcvs.2020.06.136

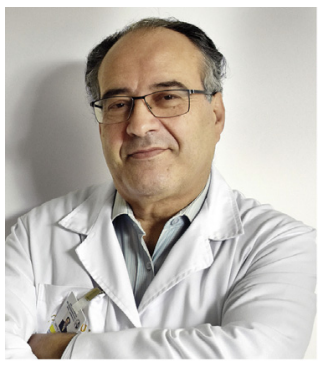

\section{REPLY: THE BAD} GUY: LEFT

\section{VENTRICULAR FUNCTION, SIZE, OR BOTH?}

\section{Reply to the Editor:}

In their article recently published in the Journal, Omer and colleagues ${ }^{1}$ demonstrated that in patients subjected to isolated coronary artery bypass grafting (CABG), depressed left ventricular ejection fraction (LVEF) was strongly associated with incremental rates of some complications (ie, renal failure, prolonged ventilation, and mechanical support), leading to increased mortality. They also found that the risk of specific complications may vary by LVEF class according to other preoperative characteristics (ie, preexisting renal failure or lung disease). They concluded that "prevention and effective treatment of complications should remain a focus of quality improvement initiatives, and future work is needed to mitigate their long-term detrimental impact on survival."

In this issue of the Journal, in a letter to the editor, Fukunaga and $\mathrm{RaO}^{2}$ draw our attention to a study from their group, published almost simultaneously in another journal, which "revealed that there was no statistically significant difference in terms of mortality, major morbidity, and prolonged hospital stay between patients with LVEF $<20 \%$ and LV size $<5.4 \mathrm{~cm}$ and those with preserved LVEF who underwent isolated CABG." In contrast, the combination of poor LV function and significant dilatation led to significantly worse outcomes. Thus, Fukunaga and Rao conclude that "the long-term effect of perioperative complications might indeed be greater in patients with LV dysfunction and an enlarged LV."
The author reported no conflicts of interest.

The Journal policy requires editors and reviewers to disclose conflicts of interest and to decline handling or reviewing manuscripts for which they may have a conflict of interest. The editors and reviewers of this article have no conflicts of interest.

Obviously, the 2 studies are not comparable; Omer and colleagues ${ }^{1}$ analyze the Veterans' Administration database involving more than 61,000 patients, including 1707 with LVEF $<25 \%$, which may be a close representation of the real world, whereas Fukunaga and Rao conducted an institutional review of a much smaller cohort (143 patients with LVEF $<20 \%$ ), which reflects the known expertise of a single institution. Nonetheless, importantly, the latter study stresses the fact that low LVEF per se is not the devil; rather, its association with ventricular dilation appears to be the reason for the poorer results. In my interpretation, low LVEF without ventricular dilation may be reversible, whereas a dilated ventricle represents a chronic phase, usually irreversible. And that makes the difference.

In an invited commentary accompanying the article by Omer and colleagues, I stressed that the relationship between preoperative comorbidities (eg, preexisting renal failure, lung disease, uncontrolled diabetes), especially when in association, and the immediate and late mortality of cardiac surgical procedures is well known. "Adding myocardial insufficiency to the 'cocktail' can only make it worse," meaning that the problem does not simply lie in the function or size of the left ventricle. Again, in my view, the main importance of the work by Omer and colleagues lies in the assumption that we can do something about reducing the number and severity of complications, thereby decreasing mortality, by modulating risk factors and modifying surgical methods and techniques. As the authors state, "prevention and effective treatment of complications should remain a focus of quality improvement initiatives, and future work is needed to mitigate their long-term detrimental impact on survival."

Manuel J. Antunes, $M D, P h D, D S c$ Faculty of Medicine Clinic of Cardiothoracic Surgery University of Coimbra Coimbra, Portugal

\section{References}

1. Omer S, Adeseye A, Jimenez E, Cornwell LD, Massarweh NN. Low left ventricular ejection fraction, complication rescue, and long-term survival after coronary artery bypass grafting. J Thorac Cardiovasc Surg. March 6, 2020; https://doi.org/ 10.1016/j.jtcvs.2020.03.040 [Epub ahead of print]. 